\title{
A Microdroplet-based Colorimetric Sensing Platform on a CMOS Imager Chip
}

Kyle R. Mallires ${ }^{\S \ddagger}$, Di Wang ${ }^{*}$, Peter Wiktor ${ }^{\ddagger}$, and Nongjian Tao ${ }^{\dagger \ddagger}$

${ }^{\S}$ School for Engineering of Matter, Transport \& Energy, Arizona State University, Tempe, Arizona 85287, United States

${ }^{\dagger}$ School of Electrical, Computer and Energy Engineering, Arizona State University, Tempe, Arizona 85287, United States

Center for Bioelectronics and Biosensors, The Biodesign Institute, Arizona State University, Tempe, Arizona, 85287, United States

Table of Contents

Page

Table S1

$\mathrm{S} 2$

Figure S1

$\mathrm{S} 2$

Figure S2

Figure S3 
Table S1. Relative Standard Deviations in Responses Across Replicate CMDs

\begin{tabular}{l|llllll} 
Ammonia (ppm) & 0.25 & 0.50 & 1.0 & 2.0 & 3.0 & 5.0 \\
\hline CMOS Imager 1 & $8.5 \%$ & $7.5 \%$ & $6.7 \%$ & $5.1 \%$ & $5.4 \%$ & $5.0 \%$ \\
CMOS Imager 2 & $9.1 \%$ & $7.7 \%$ & $6.0 \%$ & $5.8 \%$ & $5.2 \%$ & $4.8 \%$ \\
CMOS Imager 3 & $9.4 \%$ & $7.1 \%$ & $6.5 \%$ & $5.3 \%$ & $4.8 \%$ & $4.6 \%$
\end{tabular}

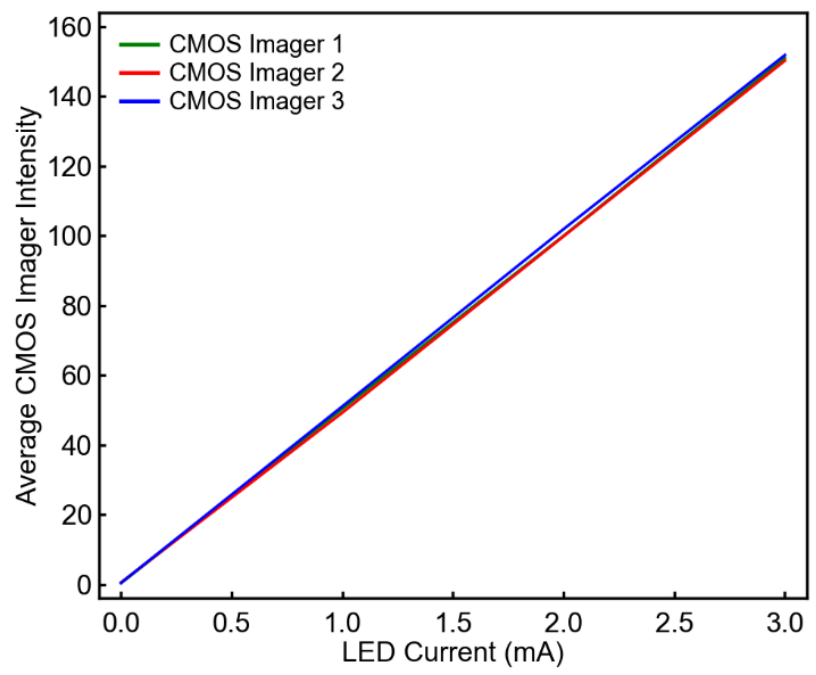

Figure S1. The average intensity of CMOS imagers 1-3 measured at 0, 1, 2, and $3 \mathrm{~mA}$ LED current. This spanned the relevant range of intensities for the CMOS imagers since experiments were carried out with the LED powered at a constant current of $2.5 \mathrm{~mA}$. The light sensitivity of the three CMOS imagers is linear and essentially identical.

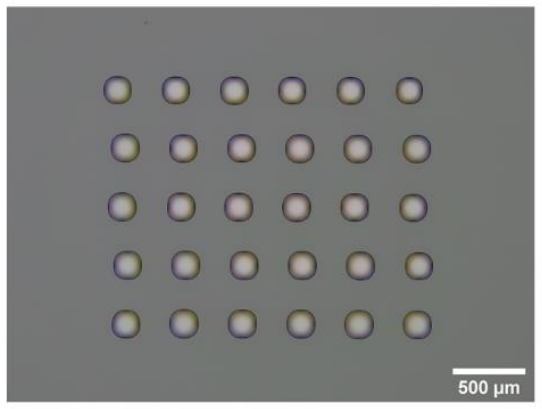

a

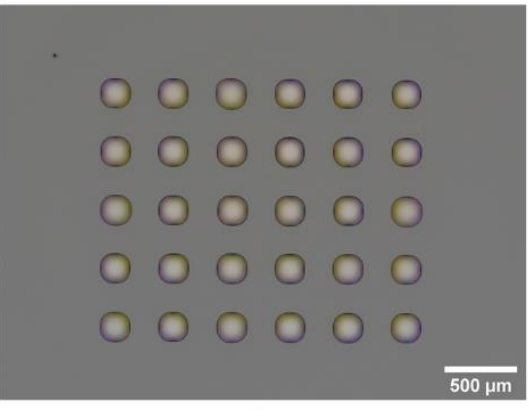

b

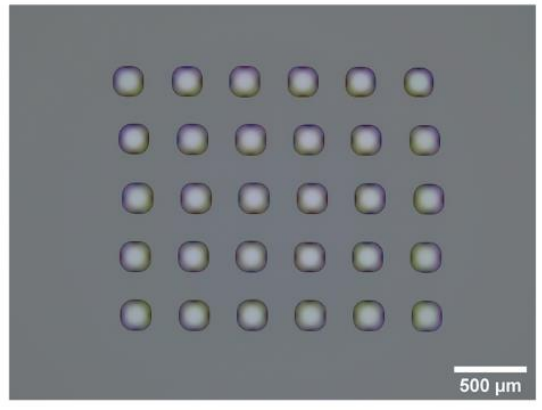

C

Figure S2. 2.4 nL CMD arrays printed on (a) CMOS Imager 1, (b) CMOS Imager 2, and (c) CMOS Imager 3. 

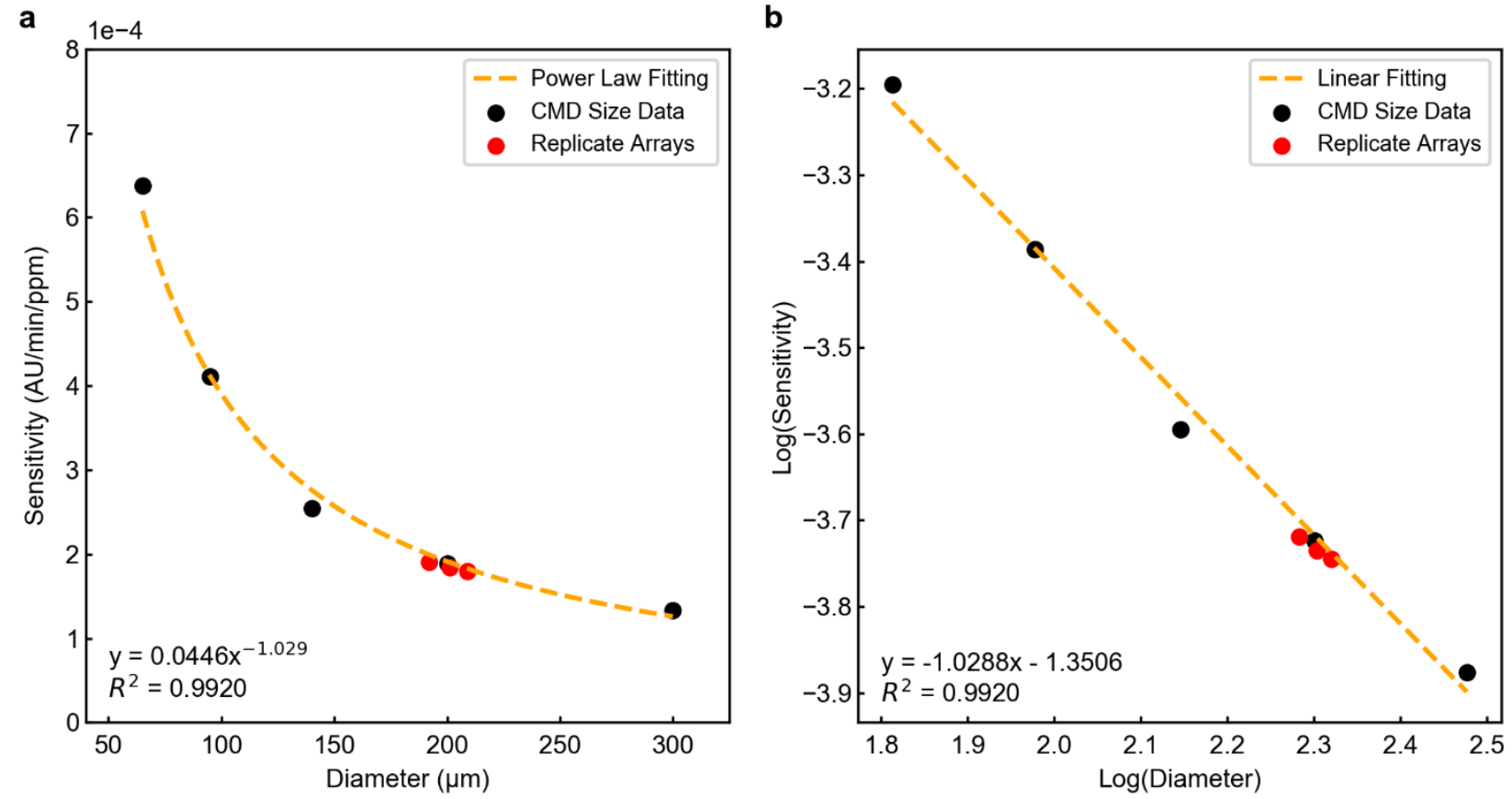

Figure S3. (a) The relationship between CMD diameter and sensitivity of the calibration curve can be modeled with a power law using data from Figure 3 and Table 1 (black dots). Sensitivities calculated during calibration of three independent CMOS imagers from Figure S2 and Table 2 lie on this line with less than 5\% error (red dots). The power law equation can be used to estimate calibration curve sensitivities (slopes) based solely on CMD diameter measured with the camera. (b) Log-log plot of sensitivity and diameter is linear suggesting that a power law may be the appropriate regression method to use in data from part (a). Indeed, the power law did provide best goodness of fit based on R-squared values compared with linear, quadratic, and exponential regressions which were 0.7592, 0.9628, and 0.9094, respectively. 